\title{
The Effects of Positron Binding and Annihilation Mechanisms in Biomolecules on PET Resolution
}

\author{
Lukáš Pichl, Member, IEEE NPSS, Masanori Tachikawa, Robert J. Buenker, Mineo Kimura, and Jan-Michael Rost
}

\begin{abstract}
The angular correlation distribution of electronpositron annihilation photons is presented for polar biological molecules, and the associated non-collinearity effect on resolution in positron emission tomography is discussed. The image resolution in PET is known to be limited by a number of factors such as radioactive decay statistics, attenuation, scatter or random coincidence of annihilation photons. This work deals with the resolution limits on the side of the measured system. Our calculated distribution of annihilation momentum belongs to the intrinsic resolution factors together with the positron range in biological tissue; the other factors are due to the interactions along the photon path from the tissue to the detector. Here we demonstrate that the angular distribution of annihilation trajectories is not necessarily gaussian, especially when the positron temporarily binds in a molecular dipole moment field before annihilation. The theoretical framework treats the electron and positron on the same footing by using the multi-reference single- and double-excitation configuration interaction algorithms applicable also to other bound-state systems. Our results form a subset of parameters relevant to the statistical image reconstruction algorithms, which may eventually increase the sensitivity of PET imaging in clinical setting.
\end{abstract}

Index Terms-PET resolution, ACAR, MRD-CI, image reconstruction, dipole-bound positron states, carbonyl group.

\section{INTRODUCTION}

$\mathbf{T}$ HE instrumentation for positron emission tomography (PET) imaging in medicine has undergone intense development over the last two decades, including the time-of-flight (TOF) techniques and various dedicated PET systems optimized for brain studies, prostate cancer detection or mammography [1]-[8]. Being superior in sensitivity to concentrations of metabolic molecules and lacking behind in image resolution,

Manuscript received July 4, 2005. This work was supported by the Japan Society for the Promotion of Science, the Japanese Ministry of Science, Sports, Culture and Education, and by the grant BU 450/7-3 of the Deutsche Forschungsgemeinschaft and the Fonds der Chemischen Industrie.

L. Pichl is now with the Max Planck Institute for the Physics of Complex Systems, Noethnitzer Str. 38, D-01187 Dresden, Germany. He is on a summer leave from the Natural Science Division, International Christian University, 3-10-2 Osawa, Mitaka, Tokyo 181-8585 Japan (e-mail: lukas@icu.ac.jp).

M. Tachikawa is with Graduate school of Science, Yokohama-city University, Seto 22-2, Kanazawa-ku, Yokohama, 236-0027 Japan (telephone: +81-45-7872188, e-mail: tachi@yokohama-cu.ac.jp).

R. J. Buenker is with Bergische Universitaet Wuppertal, Fachbereich C, Gaussstr. 20, D-42097 Wuppertal, Germany (telephone: +49-202-439-1, e-mail: buenker@uni-wuppertal.de).

M. Kimura is with the Graduate School of Sciences, Kyushu University, Fukuoka, 812-8581 Japan (e-mail: mineoscc@mbox.nc.kyushu-u.ac.jp).

J.-M. Rost is with the Max Planck Institute for the Physics of Complex Systems, Noethnitzer Str. 38, D-01187 Dresden, Germany (e-mail: rost@mpipksdresden.mpg.de).

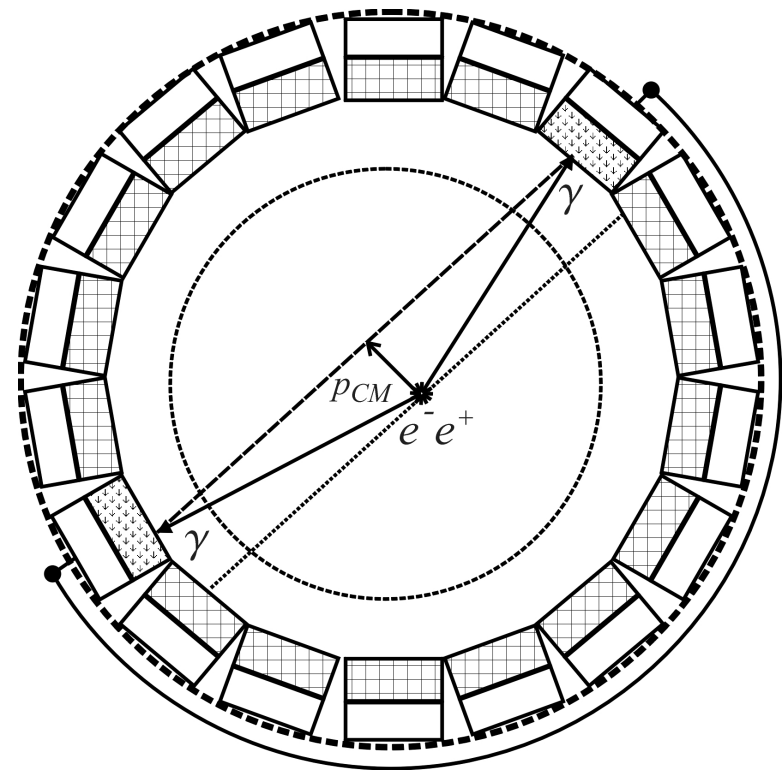

Fig. 1. Center-of-mass momentum and non-collinearity effect in PET.

PET has changed as many restrictions on the detector side and image processing were lifted, and the typical signal to noise ratio increased. Further improvements challenge understanding of annihilation mechanisms and kinetics in the biological environment of the imaged system [4], [6], [8].

Positron usually annihilates with its antiparticle, the electron, by emitting two $\gamma$ photons $(511 \mathrm{keV})$ in the opposite direction (the angle of the two photon trajectories is 180 degrees in the center-of-mass system). Annihilation into three $\gamma$ photons (totaling $1.022 \mathrm{MeV}$ and moving on less spatially restricted trajectories) is also possible but scarce; its ratio depends on how probable it is for the ortho-positronium to be formed and survive in a triplet state in the surrounding material. By measuring the coincidence of two $\gamma$ photons in the former case, the origin of the photon emitter can be located, which is the basis of all PET imaging and its medical applications. The inherent limiting factors for PET are the range of positron in matter (the length before it slows down, becomes trapped and annihilates), deflection of the annihilation photon trajectories from the straight line (due to the motion of annihilating particles in the laboratory frame), and Compton scattering (events in which the outgoing photons may loose energy and their trajectories change direction). The classical PET arrangement is sketched in Fig. 1: a circular array of crystal detectors for 
the annihilation $\gamma$ photons (these activate the charge-coupled device units denoted with arrows in Fig. 1).

The present study models the resonant trapping of positron on biomolecules by evaluating the amount of $\gamma$-ray deviation from the straight-line trajectory first. To that aim, we have extended the set of algorithms (multi-reference singleand double excitation configuration interaction, MRD-CI) for bound electron systems [9] to include the positron. Many of the experimental positron annihilation studies [10], [11], such as positron lifetime spectroscopy, Doppler-broadening spectroscopy (DOBS), or angular correlation of annihilation distribution measurements (ACAR) are performed in the solidstate phase (cf. Ref. [12] for a comprehensive account of recent development). The theoretical framework presented here is therefore considered useful for biological molecules, when the ACAR measurements are impractical or difficult.

It has been recognized that with an appropriate model of scatter and spatial effects including non-collinear trajectories, PET degradations in resolution may be largely overcome by means of signal processing [13]. The accuracy of image reconstruction is routinely increased when the time-of-flight information or photon non-collinearity is included in GEANT4 simulations [8]. In order to trace processes and objects on the level of PET resolution, the center-of-mass (CM) momentum distribution of the annihilating system should be known as precisely as possible. This in turn depends on the environment, i.e. water and a variety of organic molecules. The present work focuses on a segment of the complex process of positron annihilation, which includes in-flight annihilation (less than 5\%), positronium formation (cf. Refs. [14]-[16] for experimental results and physical models), and positron binding with intra-molecular annihilation.

The intra-molecular annihilation is a slow process, orders of magnitude above the time scale of rovibrational motion; thus the annihilation rates derive from electron-positron overlaps in space-averaged molecular configurations. For instance, Nishimura and Gianturco [17] showed that the positron lifetimes are about 20 and 500 times above the periods of the first two vibrationally excited states for $\mathrm{CH}_{4}$ and $\mathrm{C}_{2} \mathrm{H}_{2}$, respectively. This suggests a physical picture of positron annihilation in adiabatic states defined with respect to the vibrational coordinates. The equilibrium molecular distance coincides with the maximum of the ground-state vibrational wavefunction. Since the quasi-bound state of positron in urea and acetone is supported by large (over-critical) dipole moment, the positron predominantly localizes far from the molecule, and its wave function is determined to large extent by the strong dipole moment. In addition, the typical electronic density averaged over ground-state vibrational motion and the electronic density at the equilibrium nuclear geometry differ by less than about $10 \%$. Thus the electron and positron density at the equilibrium geometry represents the vibrationally-averaged quantities accurately, especially taking into account an additional averaging to be performed over all orientations of the molecule-positron complex to obtain the angular correlations. This approach has also been validated in standard quantum chemistry literature for yet another polar molecule with large dipole moment, namely the $\mathrm{LiH}-\mathrm{e}^{+}$complex [18]. For instance, Figs. 1 and 2 in Ref. [19] demonstrate that both the positron density and contact electron-positron density differ by less than 2-3\% for a bond stretch of $10 \%$, when averaged over the spatial orientation of $\mathrm{LiH}$. Rather insignificant temperature dependence in the angular correlation of annihilation photons was also found in an early theoretical study of positron annihilation in LiH diatomic crystal (Fig. 3 in Ref. [20]). Finally, even the results obtained below in this paper for two different molecules with a large dipole moment, urea and acetone, in different equilibrium geometries of different nuclei exhibit quite similar angular correlation. It should be therefore noted that the present procedure has a sound theoretical grounding for the class of biological molecules with large dipole moment. Due to the long annihilation lifetime, the positron captured by the molecule into an excited state is likely to deexcite first and deposit its excess energy to the rovibrational degrees of freedom of the molecule or in a radiative decay (see Ref. [21] for more details). High temperature of measured system may result in the importance of vibrationally excited levels. It should also be noted that positron annihilation in molecules with none or small dipole moment, unlike from the present case, is much more sensitive to nuclear geometry. ACAR distributions in solid materials generally broaden with the increasing temperature and onset of higher vibrational levels [15].

The computational procedure in this work covers the optimization of the positron-molecule $\left(\mathrm{e}^{+} \mathrm{M}\right)$ geometry, calculation of electron and positron probability distributions, and determination of space and momentum annihilation distributions. The final distribution of the $\mathrm{CM} \mathrm{e}^{+} \mathrm{e}^{-}$annihilation momentum is obtained by space averaging over all molecular orientations. By using the conservation of relativistic 4-momentum, such result directly translates into the distribution of angular deflection of annihilation photons from the straight line trajectory (cf. Fig. 1). Let us note that this deflection angle cannot be detected from the measured photons in principle in case of the $t w o-\gamma$ annihilation mechanism.

In order to assess the $\mathrm{e}^{+}$annihilation mechanism in biomolecules, we have selected two polar molecules with carbonyl group, $\mathrm{CN}_{2} \mathrm{OH}_{4}$ (urea, dipole moment 3.990D) and $\mathrm{C}_{3} \mathrm{OH}_{6}$ (acetone, dipole moment 3.263D) shown in Fig. 2. The origin of coordinates is placed at the center of mass of each molecule, which is in the proximity of the $\mathrm{C}$ atom of the carbonyl group. Typical biological tissues consist of oxygen ( $65 \%$ of body mass; water component), carbon (18.5\%), hydrogen (9.5\%), nitrogen (proteins, nucleic acids) and other chemical elements $(\mathrm{P}, \mathrm{Ca}$, $\mathrm{Fe}, \mathrm{Na}$ etc.).

Let us finally note that positron annihilation in quasibound states on molecules has long been neglected. Recently, Surko's group reported convincing experiments showing that the positron annihilation cross-sections on molecules can be enhanced by orders of magnitude as compared to scattering processes, which is due to the resonant capture of the positron in quasi-bound states [22], the major annihilation mechanism. 
These states are most easily formed when low-energy positrons vibrationally excite the target molecule by depositing their excess energy, with resonance lifetime in the order of dozens or hundreds of vibrational periods. In brief, it turns out that whenever positron can be bound by a certain molecule, there appears a series of resonances in the annihilation cross sections which dominate collisional annihilation mechanisms by orders of magnitude. This enhancement is often measured by $Z_{\text {eff }}$, the effective number of electrons against which the positron may annihilate. Resonant mechanisms yield $Z_{\text {eff }}$ much higher than the total number of electrons in the molecule. Hence our study of the importance of positron quasi-bound states, which mediate the dominant annihilation mechanism on molecules, is relevant. The two representative molecules in this work have an overcritical dipole moment, which supports an infinite number of bound states. The present case therefore does not need a vibrationally excited system, which as required e.g. for $\mathrm{CH}_{4}$ or $\mathrm{C}_{2} \mathrm{H}_{2}$ non-polar hydrocarbons. A theoretical description of the resonant process and the importance of vibrational excitations can be found e.g. in papers by Gribakin [23] and Gianturco [24].

\section{Methods}

The methods discussed in this work are (1) the image backprojection algorithm and (2) the ab initio methods to compute the photon trajectory angular distributions $f(\alpha)$ that facilitate (1). Here we focus on determining the $\alpha$-distributions, since the reconstruction algorithms have been discussed in detail in the literature [13], [25]. The present work could be in principle used in conjunction with segmentation methods for images dominated by gaussian noise [26]. Here we demonstrate the non-collinearity effect for $\mathrm{CN}_{2} \mathrm{OH}_{4}$ and $\mathrm{C}_{3} \mathrm{OH}_{6}$ in a simulated PET image reconstruction of 2D phantoms with constant and quadratic $\gamma$ source density. Atomic units (a.u.) are used throughout the paper except where mentioned otherwise.

The present quantum chemical treatment is based on ab-initio calculations of mixed positron-electron systems in adiabatic formalism by the multi-reference single- and double-excitation space configuration interaction method (MRD-CI) [9] implemented with the GRID computing technology.

\section{A. Positron binding and annihilation}

The positron is formed in-vivo by the $\beta^{+}$decay in places where the radioactive marker binds to the metabolic molecule of interest. Its kinetic energy bounded by $E_{m}$ is deposited to the tissue molecules according to Bethe-Bloch formula for $-d E / d x$ in series of ionization events. Thermalization occurs within a distance of approx. $0.2-2 \mathrm{~mm}$, depending on the positron emission energy of the particular source radioisotope (e.g. radionuclides ${ }^{11} \mathrm{C},{ }^{13} \mathrm{~N},{ }^{15} \mathrm{O}$ or ${ }^{18} \mathrm{~F}$, cf. Table 1 in Ref. [27]) and the tissue material. The probability of in-flight annihilation is below $5 \%$, since the $e^{+} / e$ annihilation cross section $\sigma_{f}$ decreases rapidly with energy. The angular distribution of annihilation photons does not depend on the $e^{+}$emitter.
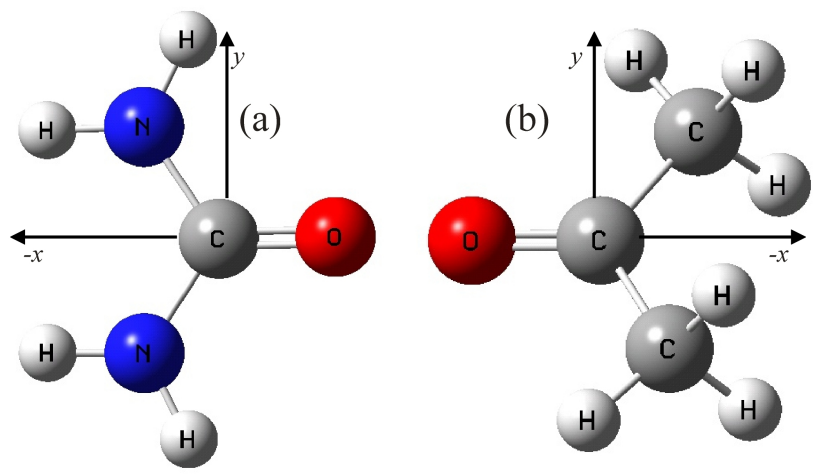

Fig. 2. Biological molecules: (a) urea (carbonyl group with amino groups) and (b) acetone (carbonyl group with methyl groups).

Positron annihilation modes other than the in-flight mechanism include direct recombination with molecular electrons or positronium (Ps) formation in ${ }^{1} S$ or ${ }^{3} S$ states, whether bound to a background molecular ion $\mathrm{M}^{+}$or not. Based on the details of the electronic structure, either bound $\mathrm{Ps} / \mathrm{M}^{+}$formation, locally bound or dipole-bound $\mathrm{e}^{+} / \mathrm{M}$ regimes occur [28].

Thermalized positron can also form positronium with free electrons created at the end of ionization path, bind at the local electron density or dipole moment potentials, or even annihilate in a direct encounter with valence electrons. Here we focus on the resonant $\mathrm{e}^{+} \mathrm{e}^{-}$annihilation mechanism in polar molecules, when the bound state is partially supported by the asymptotic dipole-moment potential. The resulting range of $\mathrm{e}^{+} \mathrm{e}^{-} \mathrm{CM}$ annihilation momentum $p_{C M}$ translates into uncertainty on the source point of the two annihilation $\gamma$ photons that are detected in coincidence during PET measurements (cf. Fig. 1).

\section{B. Ab initio calculation of $e^{+}$and $e^{-}$densities}

The wave function of the $\mathrm{e}^{-}$and $\mathrm{e}^{+}$system is found in the MRD-CI calculation with the single-positron hamiltonian,

$$
H=\sum_{i=1}^{N} h_{e}(i)+\sum_{i>j}^{N} \frac{1}{r_{i j}}+h_{p}(p)-\sum_{i=1}^{N} \frac{1}{r_{p i}},
$$

where $h_{e}(i)$ denotes the kinetic energy term of electron $i$ plus its electrostatic energy in the field of molecular nuclei; ditto $h_{p}(p)$ for positron. Electron-positron distance is labelled with $r_{p i}$ and electron-electron separation with $r_{i j}$. The total wave function is expanded in products of $N$-electron and 1positron configurations and its coefficients are found in the diagonalization of the CI matrix. Details of the theoretical procedure developed before can be found in Ref. [29].

It is also interesting to note that there exists an infinite number of $\mathrm{e}^{+}$bound states, if the molecular dipole moment (represented for simplicity by charges $\pm q$ separated by a distance $R$ ) is strong enough. The Schródinger equation for dipole-bound states is separable in the elliptic coordinates $\xi=\left(r_{+}+r_{-}\right) / R$ and $\eta=\left(r_{+}-r_{-}\right) / R$. Here $r_{ \pm}$is the electron distance from the positive and negative charge 


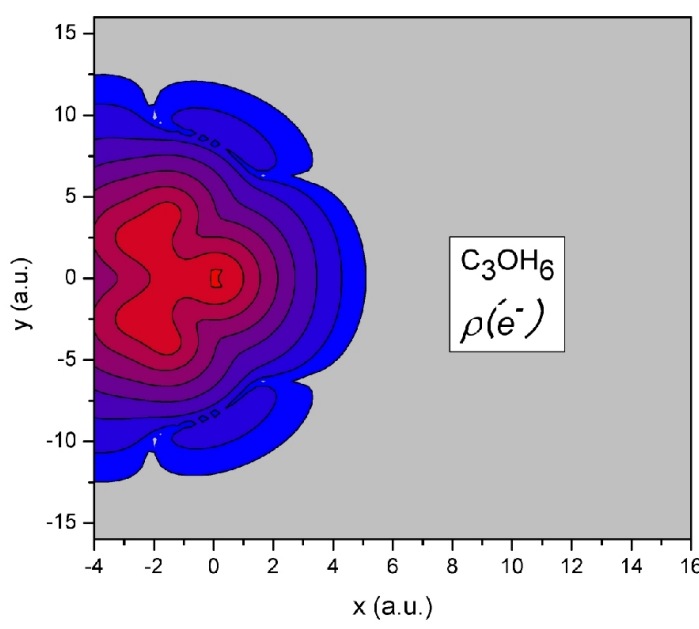

(a)

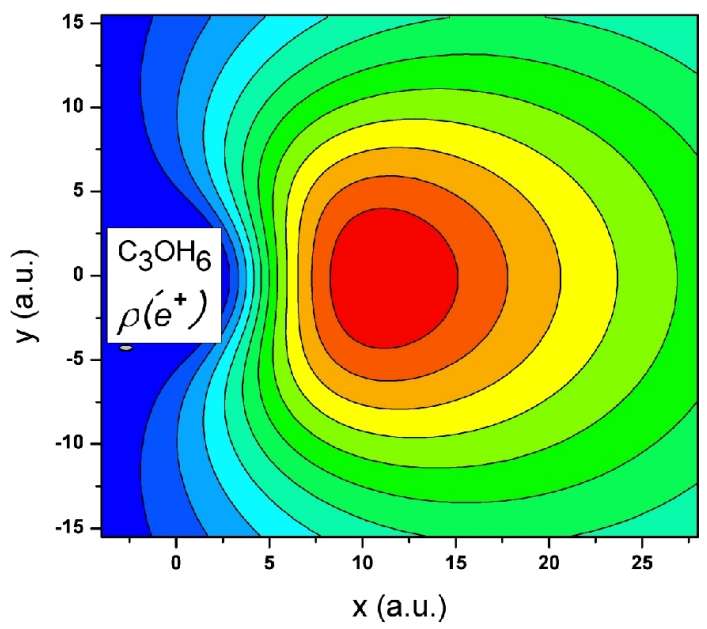

(b)
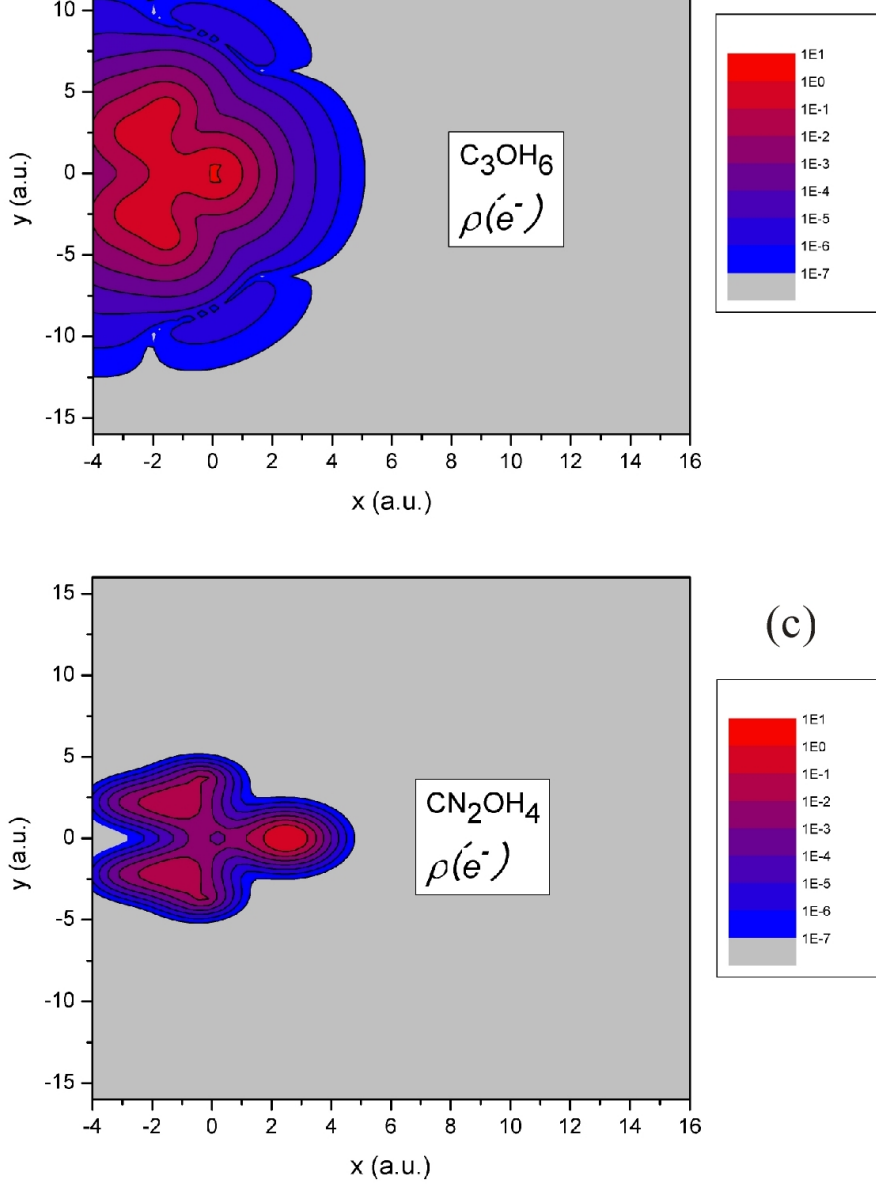

(c)
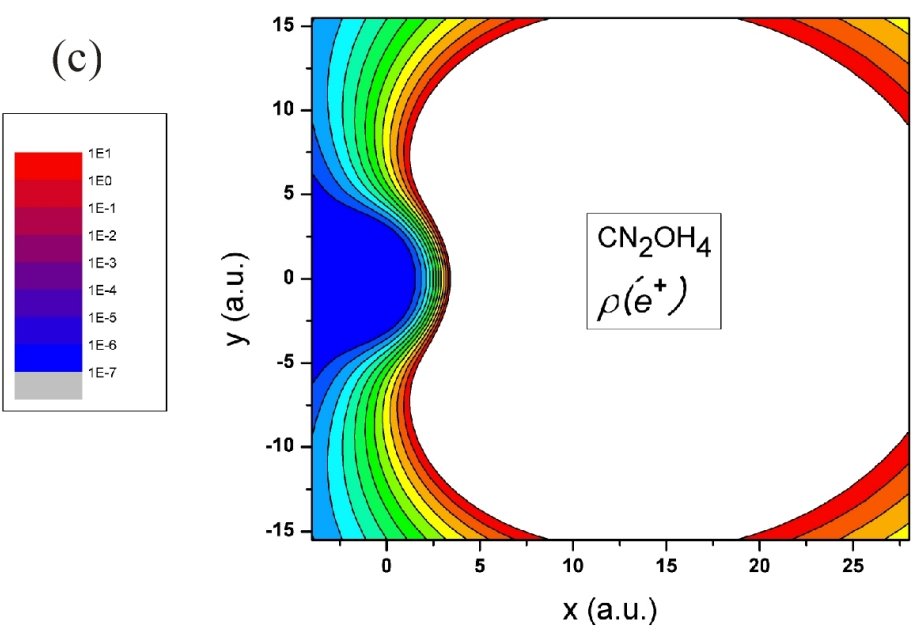

(d)

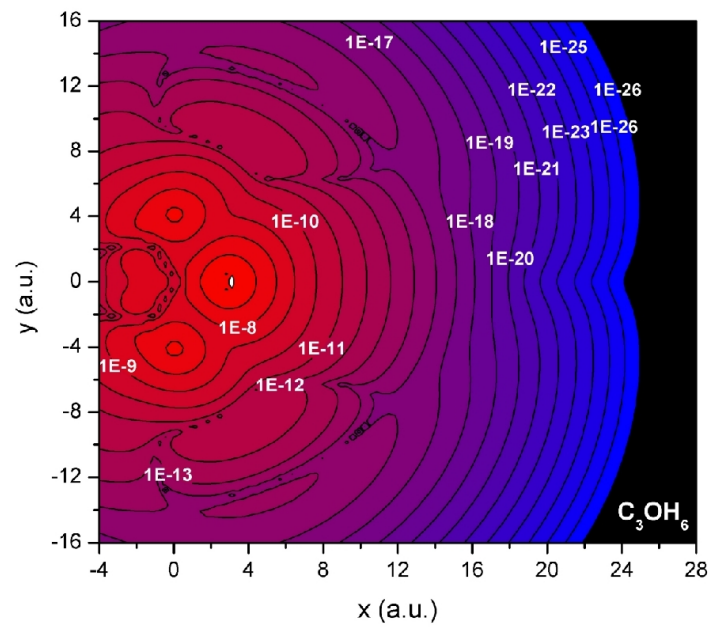

(e)

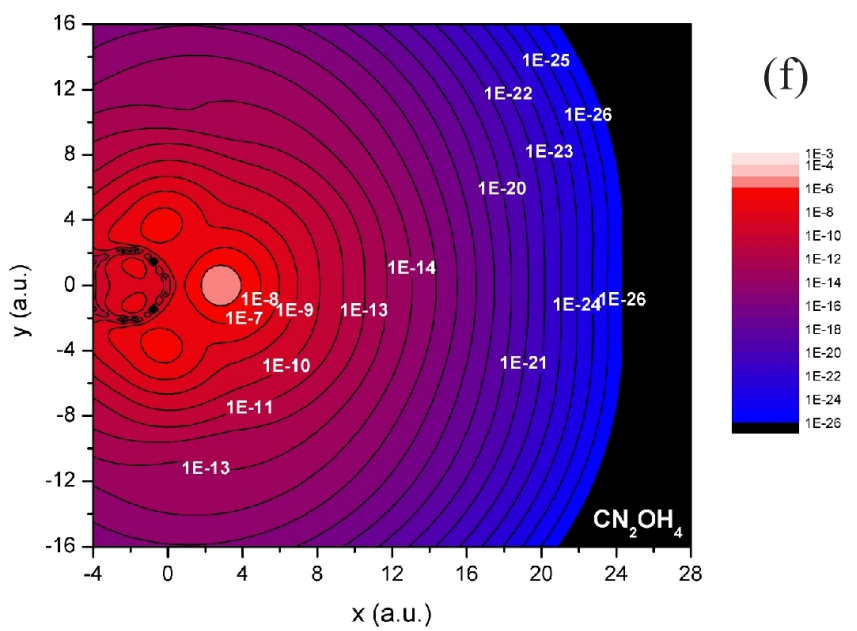

Fig. 3. Relative probabilities (in arbitrary units) for particle densities computed at CI-level: (a) Localized electron density in acetone using logarithmic scale, (b) the delocalized positron orbital in acetone, (c) electron density in urea, (d) positron orbital in urea (more compact than that of acetone), (e) simultaneous $\mathrm{e}^{+} \mathrm{e}^{-}$space annihilation density of acetone, and (d) simultaneous $\mathrm{e}^{+} \mathrm{e}^{-}$space annihilation probability density function for urea. 

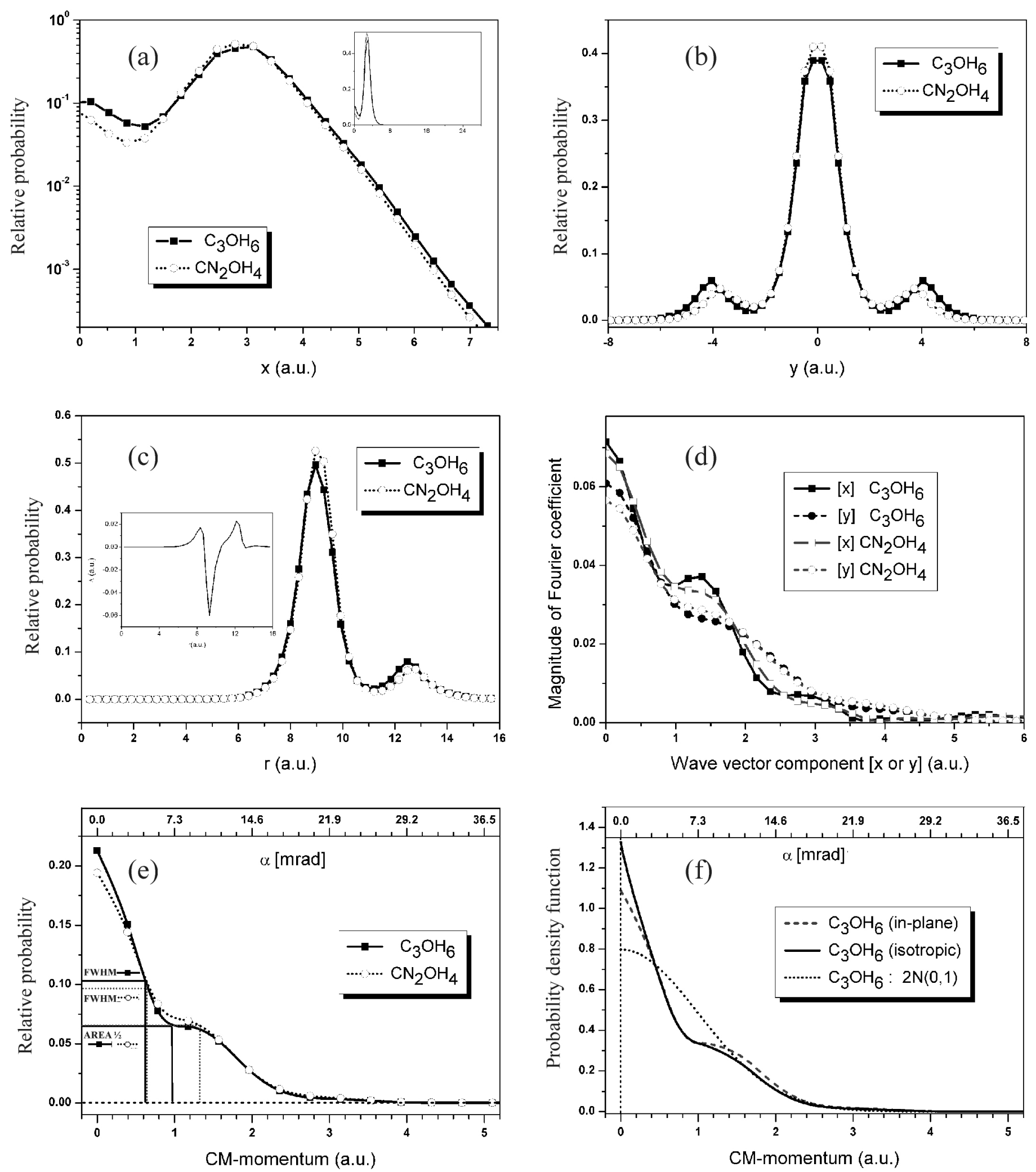

Fig. 4. Comparison of acetone and urea annihilation distributions: (a) relative annihilation probability in x-direction (inset: linear scale), (b) same as in a) except for y-direction, (c) in-plane radial annihilation probability (arbitrary units; inset: difference of acetone and urea), (d) relative wave-vector components of the annihilation packet, (e) in-plane relative CM-momentum distribution (upper scale: deflection angle; FWHM and half-area points are also indicated), (f) isotropic CM-momentum probability density function including gaussian fit as a dashed line (the integral area below the curve is normalized to $1 ; \mathrm{N}(0,1)$ in the figure stands for a normal distribution with the mean 0 and variance 1 a.u.). 

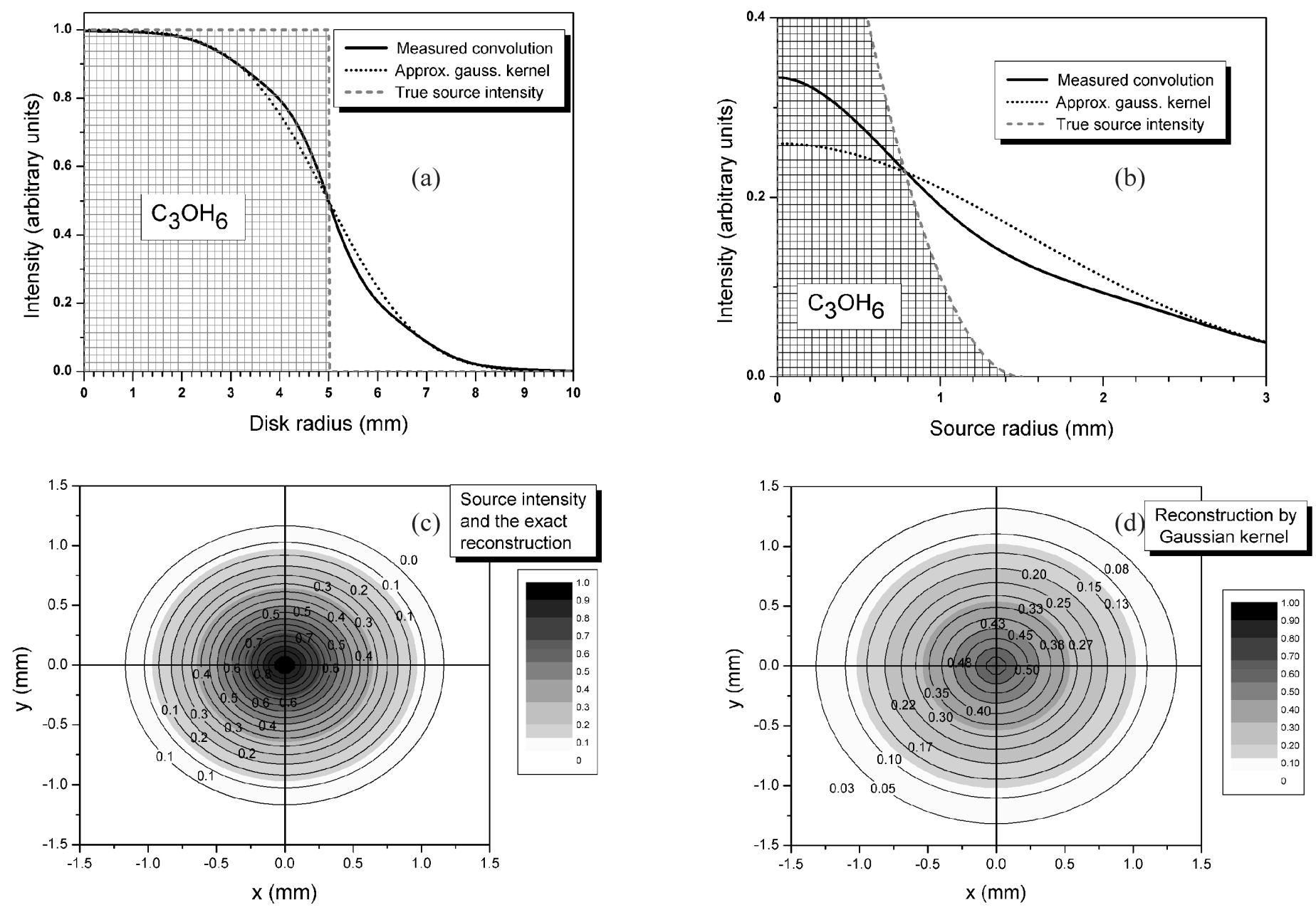

Fig. 5. Simulated 2D-phantom blurring and reconstruction (all measurement values are simulated by using the PDFs in Fig. 4f; radius of the detector circle is set as $20 \mathrm{~cm}$ ): (a) $5 \mathrm{~mm}$ step-function density source and its convolution using the exact and gaussian-approximated kernels, (b) case of source with quadratic density profile limited to $1.5 \mathrm{~mm}$ range, (c) $2 \mathrm{D}$ quadratic density source and its exact reconstruction, (d) reconstructed phantom in case c) using the approximate gaussian kernel (note the spread of density outside of the source object).

center, respectively. The total wavefunction is factored as $\varphi_{k}(\vec{r} ; R)=\varphi_{k}(\xi, \eta, \phi ; R)=C_{k}(R) X_{k}(\xi, R) Y_{k}(\eta, R) \Omega_{k}(\phi)$, where $C_{k}(R)$ is the normalization constant, and functions $X_{k}(\xi, R)$ and $Y_{k}(\eta, R)$ describe the quasi-radial and quasiangular motions of positron, subject to

$$
\begin{aligned}
& {\left[\frac{d^{2}}{d \phi^{2}}+\mu^{2}\right] \Omega(\phi)=0,} \\
& \left.\frac{d}{d \xi}\left(\xi^{2}-1\right) \frac{d}{d \xi}-\frac{\mu^{2}}{\xi^{2}-1}-c^{2}\left(\xi^{2}-1\right)+A\right] X(\xi, R)=0, \\
& \left.\frac{d}{d \eta}\left(1-\eta^{2}\right) \frac{d}{d \eta}-\frac{\mu^{2}}{1-\eta^{2}}-2 d \eta-c^{2}\left(1-\eta^{2}\right)-A\right] Y=0 .
\end{aligned}
$$

Here $d=|q| R$ stands for the dipole moment, $\mu$ labels the component of electronic angular momentum on the $\vec{R}$ axis, while $c$ and $A$ are the two remaining separation constants. In particular, $c$ relates to energy, $\epsilon=-2 c^{2} / R^{2}$ (discrete state) or $\epsilon=2 c^{2} / R^{2}$ (continuum state). Solution of the first equation reads $\Omega(\phi)=\exp (i \mu \phi) / \sqrt{2 \pi}$; the other two are readily solved in the form of power series or Legendre polynomial expansion, using either matrix-eigenvalue or continued fraction methods to determine the coefficients. In the limit $R=0$, the above equation can be easily shown to provide the critical dipole moment of $1.625 \mathrm{D}$ ( 0.639 a.u.) which supports a boundstate with zero-limit energy by definition. This critical value is common to all potentials with the same dipole-interaction asymptotics.

The probability distribution $\rho(\vec{p})$ of the $2-\gamma$ annihilation momentum is given in the form of Fourier transform of the annihilation wave function,

$$
\begin{gathered}
\rho(\vec{p})=C \int d^{3} r_{1} . . d^{3} r_{N-1} \pi\left(\vec{p} ; \vec{r}_{1} . . \vec{r}_{N-1}\right)^{2}, \\
\pi\left(\vec{p} ; \vec{r}_{1} . . \vec{r}_{N-1}\right)=\left|\int d^{3} r_{N} e^{i \overrightarrow{p_{N}}} \Psi_{p}\left(\vec{r}_{1}, \cdots, \overrightarrow{r_{N}} ; \overrightarrow{r_{N}}\right)\right|
\end{gathered}
$$


where $\Psi_{p}$ stands for the projection of the ground-state wave function $\Psi\left(\vec{r}_{1}, \cdots, \overrightarrow{r_{N}} ; \vec{R}\right)$ into the singlet state of an annihilating $e^{+} e^{-}$pair; here $N$ is the number of electrons, $C$ stands for a normalization constant, and $\vec{R}$ denotes positron coordinates. A precise derivation of the above formula is given in Ref. [28]. The space distribution of electronic and positron densities in the next section is defined by $\int|\Psi|^{2}$ where the spatial integration includes all (electron, positron) coordinates not shown explicitly.

\section{RESUlTS}

The total wave function of the ground electron-positron state is computed at the CI level using the gaussian-type correlation consistent polarized basis set augmented with diffuse functions for the electrons (aug-cc-pvdz), and a [9s5p1d] basis set which includes diffuse and polarization functions for the positron. Details of the procedure are given in Ref. [29]. Figure 3 displays the resulting electronic density for acetone (a) and urea (c) in the molecular $[x, y]$ plane. The positron cloud attaches to the oxygen atom (negative partial charge in the carboxyl $\mathrm{C}=\mathrm{O}$ group) and is more strongly localized on urea, which has a larger dipole moment (cf. Ref. [29]). The positron probability density is shown in Fig. 3b for acetone and Fig. 3d for urea, respectively. Spatial distribution of annihilation probability for acetone plotted in Fig. 3e is more diffuse than that for urea (Fig. 3f).

The $\mathrm{e}^{+} \mathrm{e}^{-}$annihilation probability resolved in either of the two in-plane directions is given in Fig. 4. The are two local maxima in the $x$-direction along the carbonyl group (Fig. $4 \mathrm{a}$, one maximum located in $x=0$ ), and three maxima in the $y$-direction (Fig. 4b), which correspond to Figs. 3a,c). Two peaks remain in the aggregate in-plane radial distribution of annihilation probability. Next, the distribution of annihilation momentum derives from the $\mathrm{e}^{+} \mathrm{e}^{-}$wave-packet components. The absolute values of the Fourier coefficients, $|\pi(\vec{p})|$ are shown in Fig. $4 \mathrm{~d}$ resolved in the in-plane $p_{x}$ and $p_{y}$ components, and the resulting distribution of $\mathrm{e}^{+} \mathrm{e}^{-} \mathrm{CM}$ annihilation momentum $\pi(|\vec{p}|)$ is plotted in Fig. 4e. The upper horizontal scale indicates the deflection angle of the annihilation trajectory in $\mathrm{mrad}$ $\left(\alpha \sim \sin \alpha \sim p_{C M} / m c\right)$. The effect is sized in the order of finestructure constant $e^{2} / 4 \pi \epsilon_{0} \hbar c$. The overall features for $\mathrm{C}_{3} \mathrm{OH}_{6}$ and $\mathrm{CN}_{2} \mathrm{OH}_{4}$ are similar, which indicates a more general trend in the dipole-bound $\mathrm{e}^{+}$systems. Let us note the tail broadening near 1 a.u. In particular, the $\alpha$ value corresponding to the FWHM is approximately $\alpha_{F W H M} \sim 4.4 \mathrm{mrad}$ for both urea and acetone, although the probability value for acetone is higher than that for urea at this point. This difference is compensated for by the more slowly decaying urea distribution. The probability density value corresponding to the median is denoted as AREA 1/2 in Fig. 4e; it is almost the same for both urea and acetone. The median angle is higher for urea than that for acetone, and also higher than $\alpha_{F W H M}$ in both cases.

Averaging over orientations of the molecular plane, we finally obtain the isotropic $\mathrm{CM}$ probability density function of annihilation momentum in Fig. 4f. Only the results for acetone are shown in Fig. 4f to decrease the number of curves; the results for urea are very similar (cf. Figs. 4a-e). The shoulder feature remains in the $\alpha$ - or $p_{C M}$-distribution. A gaussian approximation is shown in Fig. $4 \mathrm{f}$ for comparison. Its only free parameter, variance $\sigma$, is determined by equating the medians of the in-plane and approximate functions, which yields the momentum of 1 a.u. (about $8.7 \mathrm{mrad}$ ). We used the median value for the following two reasons:

1) if FWHM are equated instead, then the gaussian is too narrow and the shoulder feature in the computed distribution is completely lost; hence the interpolated gaussian fit is more reasonable;

2) the FWHM of $8.7 \mathrm{mrad}$ from median-based fit roughly corresponds to the value empirically used in PET practice. It also provides excellent reconstruction for source phantoms with uniform density, as discussed below.

In addition, it should be acknowledged that a FWHM-based gaussian fit (ignoring the shoulder in the computed distribution) would represent blurring effect of about half of the value typical for water (biological tissues).

In spite of the interpolating fit above which accounts for the shoulder feature, note how much off this kind of gaussian approximation may become, when incomplete data are retrieved from the literature and applied in image reconstruction without sufficient care. This feature is not restricted to the dipolebound positron molecular systems such as the present case; to some extent, similar behavior can be tracked e.g. in statedependent profiles of positron annihilation on atoms (cf. Fig. 8 in Ref. [28]). In both cases, the overlap of the positron with the electronic cloud is rather compact similar to the distribution of the electronic density.

The positron affinity (PA) for the two polar molecules is shown in Table I. Although it is not straightforward to estimate the sensitivity of the total annihilation rate to different PA values for molecular system (cf. Ref. [28] for atomic systems), we expect the relative distribution to maintain the shoulder feature based on the above discussions.

Having obtained the $\alpha$-distribution of trajectory deflection angles, let us proceed to phantom studies in Fig. 5. The model geometry is that of Fig. 1. The radius of the detector ring is 20 $\mathrm{cm}$ with a small 2D phantom placed in the center. The plane of the phantom source and the plane of the detector array are parallel. Because of our focus on the non-collinearity effect, image blurring due to other factors (positron range, scatter, attenuation etc.) is supposed to be described by an exact system matrix (or blurring kernel) and therefore it does not contribute to image reconstruction errors in what follows. Such a partial system matrix represents here the invertible relation between the source and the image, allowing us to focus exclusively on the additional effect. Since the non-colinearity effects come into play immediately at the annihilation time, the source object $S$ (which may be possibly blurred by positron range in matter) is transformed by an embedded function, $g(f(S))$, where $f(\cdot)$ stands for the non-colinearity effect, while $g(\cdot)$ collectively denotes all of the other blurring effects arising along the photon 
TABLE I

POSITRON BINDING ENERGIES AND POSITRON AFFINITIES

\begin{tabular}{rrrr}
\hline \hline Mol. & Basis & CI energy (a.u.) & PA (kcal/mol) \\
\hline Urea & 9s5p1d & -224.0164133 & 0.31 \\
Acetone & 9s5p1d & -191.9729683 & 0.10 \\
\hline \hline
\end{tabular}

path and in the detector.

Figure 5 illustrates the differences arising when the computed and approximate angular correlations are used in image reconstruction. First, the shaded areas in Figure 5a) and 5b) show the radial density of photon emission from simple 2D model objects (disks with constant or quadratic source density). The full lines denote the convolution of the source object with the computed angular correlation function, while the dotted lines show the convolution with the gaussian function. In particular, the source object in Fig. 5a) is a disk phantom with the radius of $5 \mathrm{~mm}$. Since the source is large in this setting and its density is uniform, blurring effects (so far without any reconstruction) resulting from both kernels are quite similar. This further justifies our particular choice of the approximate Gaussian fit. However, the situation quite changes in Fig. 5b, when a disk source with quadratic intensity is used. Now the convoluted densities obtained with the computed and approximate distribution differ more considerably, which also indicates a certain drawback hidden in the use of phantoms with only uniform source density.

Next, we proceed to image reconstruction with and without compensation. Figure $5 \mathrm{c}$ displays the disk source with quadratic intensity in 2D (the function is the same as in Fig. 5b), and its exact reconstruction using the computed $\alpha$-distribution (identical image). Finally, the reconstructed image of the phantom compensated for the approximate distribution is shown in Figure 5d. The error from the approximate kernel is substantial: should the threshold for intensity detection be about $60 \%$ of the source peak, this phantom would not be detected. Although the relative importance of such blurring due to the non-collinearity effect is lower than typical errors on the side of detector system (especially for ring detectors with small diameters), it does play an important role in PET resolution limits for mm-sized sources, along with the image processing based on phantom data and statistical reconstruction algorithms.

To complement the 2D blurring image analysis, Fig. 6 shows edge blurring for a homogenous $5 \mathrm{~mm}$ square-source phantom, demonstrating the undesirable effects of using approximate gaussian distribution. Finally, let us note that although the Figs. 5 and 6 are based only on the acetone distributions in Fig. 4e, the results for urea are very alike.

In this paper, we did not attempt to include the present results into nonlinear reconstruction algorithms, such as the nonlinear iterative ML or MAP algorithms [26]. Nevertheless, since the non-collinear effect comes to play at the origin of photon trajectories, it should be possible to partition the acollinearity effect from other blurring mechanisms and noise arising along photon trajectories and in the detector, as outlined above.
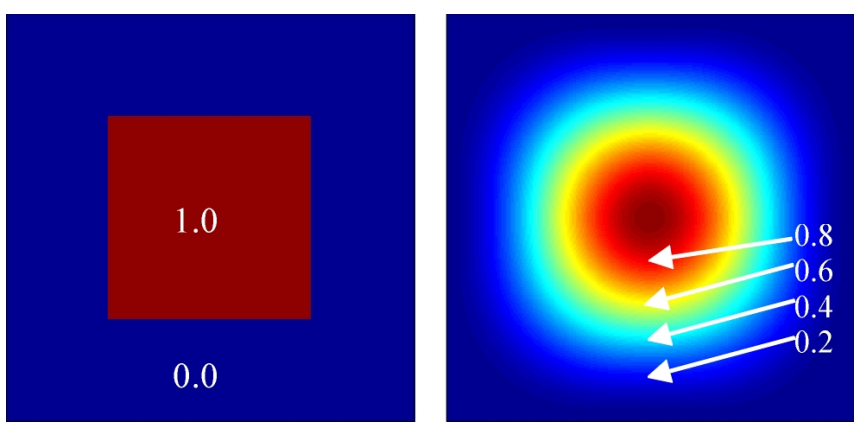

Fig. 6. Square phantom effect of lower symmetry: blurring of a square source with homogenous density (gaussian-approximated convolution kernel).

\section{CONCLUSION}

We have determined positron binding energy, electronpositron wavefunctions and the annihilation-pair center-of-mass momentum distribution for two prototype carbonyl molecules, urea and acetone, both integral and resolved in the directions of molecular plane. The distribution function is only approximately gaussian; it contains shoulders at about 2FWHM, a feature attributed to the dipole-supported positron bound state in the carbonyl group molecule. Sample phantom studies were performed using the calculated results. We have demonstrated that the difference between the accurate and approximate distribution of non-collinear trajectory angles in image reconstruction is important for the detection of low intensity sources sized about a few millimeters. A similar effect occurs also when the actual distribution consists of several superimposed gaussians but is represented only by a single gaussian component. Our results may add to the improvement of empirical approximations used in the statistical image reconstruction algorithms. The theoretical framework presented here also applies to other biological molecules with large dipole moments.

\section{REFERENCES}

[1] I. Castiglioni, O. Cremonesi, M.-C. Gilardi, A. Savi, V. Bettinardi, G. Rizzo, E. Bellotti, F. Fazio, "A Monte Carlo model of noise components in 3D PET", IEEE Trans. Nucl. Sci., vol. 49, 2002, pp. 2297-2303.

[2] M. Rafecas, G. Boning; B. J. Pichler, E. Lorenz, M. Schwaiger, S. I. Ziegler, "Effect of noise in the probability matrix used for statistical reconstruction of PET data", IEEE Trans. Nucl. Sci., vol. 51, 2004, pp. $149-156$.

[3] C. M. Laymon, R. L. Harrison, S. G. Kohlmyer, R. S. Miyaoka, T. K. Lewellen, "Characterization of single and multiple scatter from matter and activity distributions outside the FOV in 3-D PET", IEEE Trans. Nucl. Sci., vol. 51, 2004, pp. 10-15.

[4] V. Astakhov, P. Gumplinger, C. Moisan, T. J. Ruth, V. Sossi, "Effect of depth of interaction decoding on resolution in PET: A simulation study", IEEE Trans. Nucl. Sci., vol. 50, 2003, pp. 1373-1378.

[5] M. J. Schueller, T. L. Mulnix, B. T. Christian, M. Jensen, S. Holm, T. R. Oakes, A. D. Roberts, D. W. Dick, C. C. Martin, R. J. Nickles, "Addressing the third gamma problem in PET", IEEE Trans. Nucl. Sci., vol. 50, 2003, pp. 50-52.

[6] A. Welch, W. Hallett, P. Marsden, A. Bromiley, "Accurate attenuation correction in PET using short transmission scans and consistency information”, IEEE Trans. Nucl. Sci., vol. 50, 2003, pp. 427-432. 
[7] R. Lecomte, E. Croteau, M. E. Gauthier, M. Archambault, A. Aliaga, J. Rousseau, J. Cadorette, J. D. Leroux, M. D. Lepage, F. Benard, M. Bentourkia, "Cardiac PET imaging of blood flow, metabolism, and function in normal and infarcted rats", IEEE Trans. Nucl. Sci., vol. 51, 2004, pp. 696-704

[8] G. Santin, D. Strul, D. Lazaro, L. Simon, M. Krieguer, M. V. Martins, V. Breton, C. Morel, "GATE: a Geant4-based simulation platform for PET and SPECT integrating movement and time management", IEEE Trans. Nucl. Sci., vol. 50, 2003, pp. 1516-1521.

[9] L. Pichl, M. Kimura, Y. Li, and R. J. Buenker,"Branching Ratios for Secondary Processes of Water Ions Induced by Proton Beams in Radiation Therapy of Cancer", IEEE Trans. Nucl. Sci., vol. 51, 2004, pp. 1407-11.

[10] Y. Nagashima, M. Kakimoto, T. Hyodo, K. Fujiwara, A. Ichimura, T. Chang, J. Deng, T. Akahane, T. Chiba, K. Suzuki, B. T. A. McKee, A. T. Stewart, "Thermalization of free positronium atomos by collisions with silica-powder grains, aerogel grains, and gas molecules", Phys. Rev. A, vol. 52, 1995, pp. 258-265.

[11] Y. Nagai, M. Kakimoto, T. Hyodo, K. Fujiwara, H. Ikari, M. Eldrup, A. T. Stewart, "Temperature dependence of the momentum distribution of positronium in $\mathrm{MgF} 2, \mathrm{SiO} 2$, and $\mathrm{H} 2 \mathrm{O}$ ", Phys. Rev. B, vol. 62, 2000, pp. 5531-35.

[12] Positron Annihilation, edited by T. Hyodo, Y. Kobayashi, Y. Nagashima, and H. Saito, Materials Science Forum, Vols. 445-446 (Trans Tech, Aedermannsdorf, 2004).

[13] R. F. Muzic, Jr, C. H. Chen, A. D. Nelson,"A method to correct for scatter, spillover, and partial volume effects in region of interest analysis in PET", IEEE Trans. Med .Imaging., vol. 17, 1998, pp. 202-13.

[14] D. Dutta, B. Nandi Ganguly, D. Gangopadhyay, T. Mukherjee, and B. Dutta-Roy, "General trends of positronium pick-off annihilation in molecular substances", J. Phys.: Condens. Matter, vol. 14, 2002, pp. 75397549.

[15] D. Gangopadhyay, B. N. Ganguly, T. Mukherjee and B. Dutta-Roy, "A critical assessment of the bubble model for positronium annihilation in liquids", J. Phys.: Condens. Matter, vol. 11 (1999) pp. 1463-1471.

[16] M. J. Roberts, "Positronium formation in the ground and first excited states at high incident positron velocities", J. Phys. B: At. Mol. Opt. Phys., vol. 22 (1989) pp. 3315-3327.

[17] T. Nishimura and F. A. Gianturco, "Virtual-State Formation in Positron Scattering from Vibrating Molecules: A Gateway to Annihilation Enhancement", Physical Review Letters, vol. 90 (18) (2003) pp. 183201:14.

[18] K. Strasburger, "Binding energy, structure, and annihilation properties of the positron-LiH molecule complex, studied with explicitly correlated Gaussian functions", Journal of Chemical Physics, vol. 111, No. 23 (1999) pp. 10,555-558.

[19] K. Strasburger, "Adiabatic positron affinity of LiH", Journal of Chemical Physics, vol. 114, No. 2 (2001) pp. 615-616.

[20] W. Brandt, L. Edder, S. Lundqvist, "Positron Annihilation in Diatomic Crystals", Physical Review, vol. 142 (1966) pp. 165-173.

[21] P G Coleman, "Rotational excitation and momentum transfer in slow positron-molecule collisions", J. Phys. B: At. Mol. Phys., vol. 14 (1981) 2509-2517.

[22] S. J. Gilbert, L. D. Barnes, J. P. Sullivan, and C. M. Surko, “ VibrationalResonance Enhancement of Positron Annihilation in Molecules", Physical Review Letters, Vol. 88 (4) (2002) pp. 043201:1-4.

[23] G. F. Gribakin and P. M. W. Gill, "The role of vibrational doorway states in positron annihilation with large molecules", Nuclear Instruments and Methods in Physics Research B, vol. 221 (2004) pp. 30-35.

[24] F. A. Gianturco and T. Mukherjee, "Positron annihilation in $\mathrm{CO} 2$ molecules: The role of vibrational excitation", Europhysics Letters, vol. 48 (5) (1999) pp. 519-525.

[25] T. E. Nichols, J. Qi, E. Asma, R. M. Leahy, "Spatiotemporal reconstruction of list-mode PET data", IEEE Trans. Med. Imaging, vol. 21 (2002) pp. 396-404.

[26] Z. W. Bell, "A Bayesian/Monte Carlo Segmentation Method for Images Dominated by Gaussian Noise", IEEE Transactions on Pattern Analysis and Machine Intelligence, vol. 11 (9) (1989) pp. pp. 985-990.

[27] J. A. K. Blokland, P. Trindev, M. P. M. Stokkel, E. K. J. Pauwels, "Positron emission tomography: a technical introduction for clinicians", European Journal of Radiology, vol. 44 (2002) pp. 70-75.

[28] J. Mitroy, M. W. J. Bromley, and G. G. Ryzhikh, "Positron and positronium binding to atoms", J. Phys. B, vol. 35 (2002) pp. R81-R116.
[29] M. Tachikawa, R. J. Buenker, and M. Kimura,"Bound states of positron with urea and acetone molecules using configuration interaction $a b$ initio approach", J. Chem. Phys., vol. 119 (2003) pp. 5005-5009. 